OPEN ACCESS

Edited by:

Vinod Tiwari,

Indian Institute of Technology (BHU),

India

Reviewed by:

Ningjian Wang,

Shanghai Jiao Tong University, China

Kok Yong Chin,

National University of Malaysia,

Malaysia

*Correspondence:

Manna Zhang

mannazhang@126.com

${ }^{t}$ These authors have contributed equally to this work

Specialty section: This article was submitted to Clinical Diabetes, a section of the journa Frontiers in Endocrinology

Received: 03 March 2021 Accepted: 14 May 2021

Published: 31 May 2021

Citation:

Cai M, Cui R, Yang P, Gao J, Cheng $X$, Sheng C, Li H, Sheng H, Qu S and Zhang M (2021) Incidence and Risk Factors of Hypogonadism in Male Patients With Latent Autoimmune Diabetes and Classic Type 2 Diabetes.

Front. Endocrinol. 12:675525. doi: 10.3389/fendo.2021.675525

\section{Incidence and Risk Factors of Hypogonadism in Male Patients With Latent Autoimmune Diabetes and Classic Type 2 Diabetes}

\author{
Meili Cai ${ }^{\dagger}$, Ran Cui ${ }^{\dagger}$, Peng Yang ${ }^{\dagger}$, Jingyang Gao, Xiaoyun Cheng, Chunjun Sheng, \\ Hong Li, Hui Sheng, Shen Qu and Manna Zhang* \\ Department of Endocrinology \& Metabolism, Shanghai Tenth People's Hospital, Tongji University School of Medicine, \\ Shanghai, China
}

Objectives: This study aimed to compare the prevalence of hypogonadism between male patients with latent autoimmune diabetes (LADA) and type 2 diabetes (T2DM) and investigate the risk factors for hypogonadism in these patients.

Methods: This cross-sectional study evaluated 367 male patients with LADA ( $n=73)$ and T2DM ( $n=294)$ who visited the endocrinology department of Shanghai Tenth People's Hospital between January 2016 and October 2019 for diabetes management. Sex hormones, lipid profiles, sex hormone-binding globulin (SHBG), glycosylated hemoglobin A1c, beta-cell function, uric acid, and osteocalcin were determined in serum samples. Hypogonadism was defined as calculated free testosterone (cFT) less than $220 \mathrm{pmol} / \mathrm{L}$ along with the presence of symptoms (positive ADAM score).

Results: The rate of hypogonadism in the LADA and T2DM group were 8.2, and 21.7\%, respectively ( $p=0.017$ ). After adjusting possible confounders, the rate of hypogonadism in the LADA group was comparable to those of the T2DM group. Univariate logistic regressions demonstrated that age, BMI, fasting C-peptide, triglycerides, total cholesterol and uric acid were associated with hypogonadism in men with diabetes, $\mathrm{BMI}$, triglycerides and estradiol were independent risk for hypogonadism in men with diabetes.

Conclusion: This is the first evidence to explore the rate of hypogonadism in male patients with latent autoimmune diabetes ( $\angle A D A)$. In the population requiring admission to a large urban hospital in China, the rate of hypogonadism was comparable to those of the T2DM group after adjusting for possible confounders. BMl, triglycerides and estradiol were independently associated with the presence of $\mathrm{HH}$ in male diabetic patients.

Keywords: hypogonadism, type 2 diabetes, adult latent autoimmune diabetes (LADA), low testosterone in men with diabetes, risk factors 


\section{INTRODUCTION}

Latent autoimmune diabetes in adults (LADA) is a highly heterogeneous disease and the most common form of adultonset autoimmune diabetes. Patients with LADA initially do not require insulin but eventually become insulin dependent after a rapid decline in beta cell mass and function $(1,2)$. Many epidemiological studies have shown that the prevalence of LADA was 3.3- to 12.2-fold higher than that of adult-onset type 1 diabetes (T1DM), with LADA accounting for $1.5 \%$ to $14 \%$ of patients previously diagnosed with type 2 diabetes (T2DM) (3). These findings suggest the important role of LADA in the wide spectrum of diabetes.

Hypogonadism is characterized by low concentrations of testosterone and is widely associated with insulin resistance, diabetes, obesity, and metabolic syndrome. Increasing evidence has shown that the high prevalence of hypogonadism in male patients with T2DM and testosterone supplement could improve glucose metabolism and insulin sensitivity in those patients (4-6). There is a bidirectional relationship between hypogonadism and diabetes. For one thing, testosterone could up-regulate the expression of glucose transporter 4 (GLUT4) and insulin receptor substrate 1 to stimulate glucose uptake into muscle and adipose (7); for another, insulin resistance, inflammatory mediators and increased aromatase activity could reduce LH concentrations, resulting in decreased testosterone (8). Although there have been reports of hypogonadism in male patients with classical T1DM $(9,10)$, no study has evaluated the incidence rate of hypogonadism in male patients according to the specific types of diabetes. Previous studies reported that many clinical features in LADA are continuous spectrums between classical T1DM and T2DM $(1,2)$, for instance, the prevalence of metabolic syndrome in the patients with LADA fell in between classical T1DM and T2DM $(11,12)$; the prevalence of cardiac autonomic neuropathy and osteoporosis in LADA were comparable to T1DM but different from $\operatorname{T2DM}(13,14)$. Thus, it is important to investigate the clinical characteristic of hypogonadism in patients with LADA.

Accordingly, this study aimed to compare the occurrence rate of hypogonadism among male patients with diabetes according to its specific type(LADA, and classical T2DM) and investigated the risk factors for hypogonadism in men with diabetes, which would help us to explore the association of hypogonadism and the etiology of hyperglycemia.

\section{PATIENTS AND METHODS}

\section{Study Design and Patients}

This cross-sectional study was approved by the Institutional Human Subjects Review Board of Shanghai Tenth People's Hospital. Written informed consent was obtained from all participants. We evaluated male patients with diabetes who visited the Endocrinology Department of Shanghai Tenth People's Hospital between January 2016 and October 2019 for better control and management of diabetes. Patients with moderate or severe liver and renal dysfunction, with history of congenital/hypogonadotropic hypogonadism, hyperthyroidism, varicocele, epididymitis, testicle injury, abnormal karyotype, or those who received testosterone replacement therapy in the past 6 months were excluded. The cohort involved 367 patients; of them, 73 patients had LADA; and 294 patients, T2DM.

\section{Diagnosis and Measurements}

The diagnostic criteria for LADA were glutamic acid decarboxylase (GAD) antibody-positivity, initially non-insulin requiring for at least 6 months, and diagnosed over the age of 30 years (15). Classical T2DM were diagnosed according to the criteria set by the World Health Organization. The androgen deficiency in the aging male (ADAM) questionnaire was completed by the patient with low testosterone levels $(\mathrm{cFT}<220$ $\mathrm{pmol} / \mathrm{L}$ ). Hypogonadism was defined as calculated free testosterone (cFT) less than $220 \mathrm{pmol} / \mathrm{L}$ along with the presence of symptoms (positive ADAM score) $(16,17)$. It was further classified as primary hypogonadism and hypogonadotropic hypogonadism according to the levels of luteinizing hormone (LH) and follicle-stimulating hormone (FSH). Specifically, if the level of LH or FSH was less than 10 IU/L, the patient was diagnosed with hypogonadotropic hypogonadism; otherwise, the patient will be diagnosed with primary hypogonadism (18).

The participant's medical history, body mass index (BMI), and hypertension were recorded. Blood samples were obtained from all patients in the morning after at least 10 hours of overnight fasting. Fasting plasma glucose, fasting C-peptide, glycosylated hemoglobin A1c (HbA1c), GAD antibodies, creatinine, uric acid, total calcium, total cholesterol, triglyceride, high-density lipoprotein cholesterol, and lowdensity lipoprotein cholesterol (LDL-c) were measured. A standard meal load test ( $70 \mathrm{~g}$ of instant noodles equivalent to an energy intake of 500 kilocalories) was performed, followed by examination of plasma glucose and C-peptide in $120 \mathrm{~min}$ (19). The total testosterone, estradiol, LH, FSH and SHBG were determined by the electrochemiluminescence immunoassay [(Roche Diagnostics GmbH, Cot. Germany). Testosterone: intra- \& interassay CV $\leq 1.2 \%-18.1 \%$, lower limit of detection: $0.025 \mathrm{ng} / \mathrm{ml}$, measurement range: $0.025-15 \mathrm{ng} / \mathrm{ml}$; LH: intra- \& interassay $\mathrm{CV} \leq 0.8 \%-1.8 \%$, lower limit of detection: $<0.100$ $\mathrm{mIU} / \mathrm{mL}$, measurement range: $0.100-200 \mathrm{mIU} / \mathrm{mL}$; FSH, intra- \& interassay $\mathrm{CV} \leq 1.3-2.8 \%$, lower limit of detection: $0.100 \mathrm{mIU} /$ $\mathrm{mL}$, measurement range: $>0.100-200 \mathrm{mIU} / \mathrm{mL}]$. Serum osteocalcin (OC) was tested using an N-MID osteocalcin enzyme-linked immunosorbent assay kit (Elecsys, Roche diagnostic Ltd., Switzerland). Sartorius et al.'s formula $\left(\mathrm{cFT}=24.00314 \mathrm{xT} / \log 10\right.$ (SHBG)-0.04599 $\mathrm{xT}^{2}$ ) was adopted to calculate free testosterone concentrations $(20,21)$.

The microvascular disease was defined as presence of macroalbuminuria, diabetic neuropathy and diabetic retinopathy. The macrovascular disease was defined as presence of cerebrovascular disease, coronary heart disease, heart failure, or peripheral arterial disease. Plaques were identified by the ultrasonography test, including the common carotid artery, superficial carotid artery, common femoral artery 
and superficial femoral artery. The above data were collected by reviewing of all patient's electronic medical records.

\section{Statistical Analysis}

Continuous data were compared among the two groups using linear regression analyses. Meanwhile, categorical data were compared among the three groups using logistic regression analyses. Multiple logistic regression models were used to determine the effect of diabetes types on the diagnosis of hypogonadism and investigate the independent risk factors of hypogonadism. Variables with $\mathrm{P}<0.20$ in univariate analysis were included in the multiple logistic regression analyses. All statistical analyses were performed using SPSS version 20.0 software. A P value of $<0.05$ was considered statistically significant.

\section{RESULTS}

The mean age of the patients with LADA and T2DM was $53.6 \pm$ 13.2 and $51.9 \pm 16.3$ years, respectively. Considering the BMI was significantly different in two groups, the comparison of clinical features between them was adjusted by BMI. The LADA group had lower SBP than the T2DM group. The mean fasting cpeptide, postprandial c-peptide, uric acid and triglyceride levels in the LADA group were lower than those of the T2DM group. Meanwhile, the HDL-c levels in the LADA group were higher than those of the T2DM group. The mean DBP, total cholesterol, LDL-c, creatinine and osteocalcin levels were similar between the two groups. The frequencies of microvascular disease, macrovascular disease and plaques were comparable between the two groups (Table 1). There were no significant differences in $\mathrm{LH}, \mathrm{FSH}$ and $\mathrm{cFT}$ levels between the two groups. The mean testosterone, $\mathrm{E}_{2}$ and SHBG levels of the LADA group were different from those of the T2DM group.

The rate of hypogonadism was much lower in the LADA group than the T2DM group $(8.2 \%$ vs $21.7 \%, \mathrm{P}=0.017)$. The incidence rates of hypogonadotropic hypogonadism in the LADA and T2DM groups were $6.8 \%, 17.6 \%$, respectively $(\mathrm{P}=0.019)$. Meanwhile, the incidence rates of primary hypogonadism in the LADA and T2DM groups was $1.4 \%$ and $4.1 \%$, respectively $(\mathrm{P}=0.318)$ (Figure 1$)$. To determine the influence of diabetes types along with confounders on the diagnosis of hypogonadism, multiple logistic models were used to compare the rate of hypogonadism between two groups. After adjusting possible confounders (Model 3 and Model 4), the rate of hypogonadism in the LADA group was comparable to those of the T2DM group (Table 2).

The risk factors for hypogonadism in male diabetic patients was explored in the overall population. Univariate logistic

TABLE 1 | General characteristics of male patients with LADA and T2DM.

\begin{tabular}{|c|c|c|c|}
\hline Items & $\begin{array}{r}\text { LADA } \\
(\mathrm{N}=73)\end{array}$ & $\begin{array}{c}\text { T2DM } \\
\text { (N=294) }\end{array}$ & P Values \\
\hline Age (years) & $53.6 \pm 13.2$ & $51.9 \pm 16.3$ & 0.930 \\
\hline Age at diagnosis (years) & $43.0 \pm 11.7$ & $42.9 \pm 13.5$ & 0.823 \\
\hline Duration of diabetes (years) & $10.0 \pm 8.5$ & $10.4 \pm 7.4$ & 0.150 \\
\hline Family history of diabetes $(\%, n)$ & $50.7(37)$ & $46.4(136)$ & 0.880 \\
\hline Smoking (\%,n) & $54.8(40)$ & $36.5(107)$ & 0.023 \\
\hline $\mathrm{BMl}\left(\mathrm{kg} / \mathrm{m}^{2}\right)$ & $20.8 \pm 2.2$ & $25.9 \pm 4.1$ & $<0.001$ \\
\hline $\mathrm{SBP}(\mathrm{mmHg})$ & $126.4 \pm 19.6$ & $136.1 \pm 18.2$ & 0.047 \\
\hline $\mathrm{DBP}(\mathrm{mmHg})$ & $73.2 \pm 10.6$ & $78.4 \pm 12.3$ & 0.098 \\
\hline $\mathrm{HbA1c}(\%)$ & $10.4 \pm 2.3$ & $9.3 \pm 2.3$ & 0.599 \\
\hline Fasting serum C-peptide(ng/mL) & $0.2 \pm 0.2$ & $2.1 \pm 1.3$ & $<0.001$ \\
\hline Postprandial C-peptide (ng/mL) & $0.4 \pm 0.4$ & $4.8 \pm 3.1$ & $<0.001$ \\
\hline Serum creatinine (umol/L) & $68.1 \pm 15.5$ & $71.6 \pm 49.1$ & 0.166 \\
\hline Uric acid (umol/L) & $263.6 \pm 95.7$ & $352.9 \pm 113.7$ & 0.003 \\
\hline Total cholesterol (mmol/L) & $4.4 \pm 1.7$ & $4.6 \pm 1.4$ & 0.239 \\
\hline Triglycerides (mmol/L) & $0.8(0.6-1.3)$ & $1.5(1.0-2.3)$ & 0.016 \\
\hline HDL-c (mmol/L) & $1.3 \pm 0.3$ & $1.0 \pm 0.3$ & 0.003 \\
\hline LDL-c (mmol/L) & $2.5 \pm 1.5$ & $2.7 \pm 1.0$ & 0.205 \\
\hline Osteocalcin (ng/ml) & $12.1 \pm 5.1$ & $12.8 \pm 6.1$ & 0.270 \\
\hline Microvascular disease (\%) & $41.1(30)$ & $47.6(139)$ & 0.327 \\
\hline Macrovascular disease (\%) & $20.5(15)$ & $28.6(84)$ & 0.315 \\
\hline Plaques (\%) & $53.4(39)$ & $48.3(142)$ & 0.815 \\
\hline LH (IU/L) & $7.6 \pm 4.2$ & $7.6 \pm 4.8$ & 0.601 \\
\hline FSH (IU/L) & $8.6 \pm 6.1$ & $9.4 \pm 6.3$ & 0.532 \\
\hline $\mathrm{E}_{2}(\mathrm{pmol} / \mathrm{L})$ & $104.6 \pm 53.8$ & $108.2 \pm 48.5$ & 0.004 \\
\hline Testosterone (ng/ml) & $6.1 \pm 2.2$ & $4.0 \pm 1.9$ & 0.001 \\
\hline $\mathrm{SHBG}(\mathrm{nmol} / \mathrm{L})$ & $62.6 \pm 15.9$ & $34.9 \pm 23.2$ & 0.028 \\
\hline $\mathrm{cFT}(\mathrm{nmol} / \mathrm{ml})$ & $263.0 \pm 134.6$ & $204.8 \pm 94.7$ & 0.340 \\
\hline
\end{tabular}

BMI, body Mass Index; SBP, systolic blood pressure; DBP, diastolic blood pressure; HbA1C, glycosylated hemoglobin A1C; HDL-C, high-density lipoprotein cholesterol; LDL-C, lowdensity lipoprotein cholesterol; LH, luteinizing hormone; FSH, follicle-stimulating hormone; $E_{2}$, Estradiol; SHBG, sex hormone-binding globulin cFT; calculated free testosterone. All data were adjusting for BMI.

The bold values indicates statistical significance. 
A

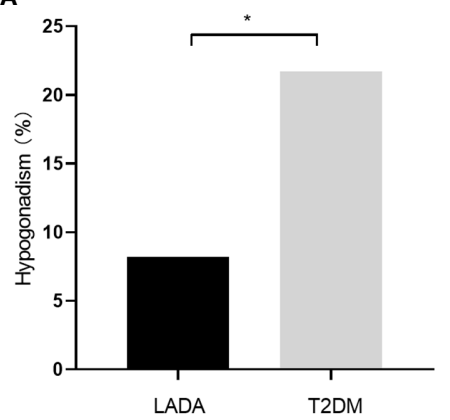

B

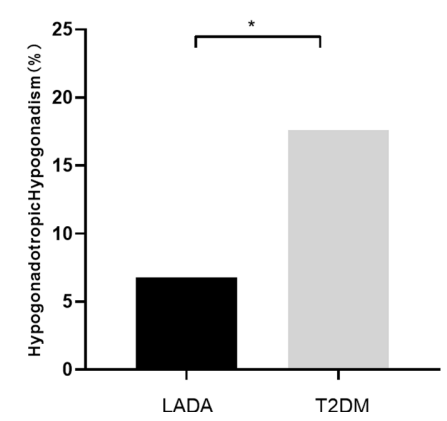

c

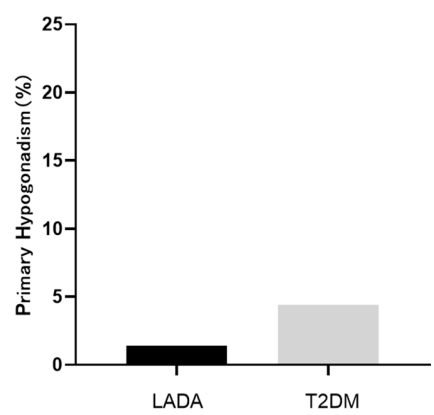

FIGURE 1 | (A) The incidence rates of hypogonadism in the LADA and T2DM groups (8.2\% vs 21.7\%, P=0.017); (B) The incidence rates of hypogonadotropic hypogonadism in the LADA and T2DM groups (6.8\% vs 17.6\%, P=0.019); (C) The incidence rates of primary hypogonadism in the LADA and T2DM groups (1.4\% vs $4.1 \%, P=0.318)$. The symbol "*” indicates statistical significance.

TABLE 2 | Multiple logistic regression of hypogonadism and type of diabetes.

\begin{tabular}{|c|c|c|c|c|}
\hline \multirow[t]{2}{*}{ Rate of hypogonadism } & \multirow{2}{*}{$\begin{array}{c}\text { Reference } \\
\text { (LADA) }\end{array}$} & \multicolumn{3}{|c|}{ Type 2 Diabetes } \\
\hline & & OR & $95 \% \mathrm{Cl}$ & P Values \\
\hline Model 1 & 1 & 3.061 & 1.172-7.994 & 0.022 \\
\hline Model 2 & 1 & 2.942 & 1.115-7.759 & 0.029 \\
\hline Model 3 & 1 & 2.289 & $0.738-7.093$ & 0.151 \\
\hline Model 4 & 1 & 1.932 & $0.690-5.406$ & 0.209 \\
\hline
\end{tabular}

OR, odds ratio Cl; confidence interval; Model 1 was unadjusted; Model 2 was adjusted for age, duration of diabetes and smoking; Model 3 was adjusted for age, duration of diabetes, smoking, family history, BMI, hypertension; Model 4 was adjusted for age, duration of diabetes, smoking, family history, BMI, hypertension, HbA1C, creatinine, triglycerides.

The bold values indicates statistical significance.

regressions demonstrated that age, BMI, fasting C-peptide, triglycerides, total cholesterol and uric acid were associated with hypogonadism in men with diabetes (Table 3). Moreover, BMI (OR 1.149 [95\% CI: 1.033-1.279]), triglycerides (OR 1.167 [95\% CI:1.014-1.343]) and estradiol (OR 0.96 [95\% CI:0.9760.995]) were independently associated with the presence of hypogonadism in the multiple logistic regression analyses. There were no association between hypogonadism status and HbA1c, fasting glucose, osteocalcin, microvascular or macrovascular complications of diabetes, and presence of plaques (Table 4).

\section{DISCUSSION}

Increasing evidence has shown that hypogonadism was associated with insulin resistance, diabetes, obesity, and metabolic syndrome $(4,6)$. Moreover, the rate of hypogonadism is higher in patients with T2DM but lower in patients with classical T1DM $(5,9,10)$. However, studies on the rate of hypogonadism in patients with LADA are limited. Given that the clinical characteristics of LADA overlaps both T1DM and T2DM, it is important to investigate testosterone levels in patients with LADA. The study would help us to explore the association of hypogonadism and the etiology of hyperglycemia.
In our study, the mean total testosterone levels in patients with LADA were significantly different from those in patients with T2DM. This was consistent with previous studies that testosterone levels tended to be normal in patients with T1DM but lower in patients with $\operatorname{T2DM}(9,10)$. Given the much larger BMI of the men in the T2DM group, a low free $\mathrm{T}$ along with positive ADAM questionnaire were using as the threshold to "diagnose" hypogonadism in the present study. The rates of hypogonadism in patients with T2DM was $21.7 \%$, which were generally in line with previous studies showing that hypogonadism was prevalent in $25 \%-40 \%$ of patients with T2DM $(22,23)$. It is interesting to note that compared with T2DM, patients with LADA had higher SHBG level but lower rate of hypogonadism. Previous studies have shown that obesity could aggravate low-grade inflammation, resulting in low levels of SHBG and testosterone $(24,25)$. Considering BMI was markedly low in the LADA group, our results might be explained by the fact that the effects of obesity on testosterone are more substantial than the effects of SHBG on testosterone in diabetic men. After controlling for confounders in multiple logistic regression models, the discrepancy between the LADA and T2DM groups was disappeared. This result indicates that the association of hypogonadism status and the etiology of hyperglycemia was limited. This result needs to be validated in studies with large sample sizes. 
TABLE 3 | Univariate logistic regressions between variables and hypogonadism status.

\begin{tabular}{|c|c|c|c|}
\hline Variable & OR & $95 \% \mathrm{Cl}$ & P Values \\
\hline Age & 1.127 & $1.057-1.324$ & 0.016 \\
\hline $\mathrm{BMl}$ & 1.119 & 1.116-1.229 & $<0.001$ \\
\hline Fasting Glucose & 1.026 & 0.957-1.102 & 0.461 \\
\hline Fasting C-peptide & 1.397 & 1.147-1.704 & 0.001 \\
\hline $\mathrm{HbA1c}$ & 1.004 & $0.890-1.131$ & 0.952 \\
\hline Triglycerides & 1.242 & $1.084-1.423$ & 0.002 \\
\hline Total cholesterol & 1.223 & $1.010-1.479$ & 0.039 \\
\hline HDL-C & 0.649 & $0.281-1.499$ & 0.311 \\
\hline LDL-C & 0.921 & $0.686-1.236$ & 0.582 \\
\hline Uric acid & 1.003 & $1.002-1.005$ & 0.020 \\
\hline Osteocalcin & 0.945 & $0.889-1.004$ & 0.066 \\
\hline$E_{2}$ & 0.995 & $0.989-1.001$ & 0.128 \\
\hline Microvascular disease & 1.173 & $0.593-2.215$ & 0.532 \\
\hline Macrovascular disease & 1.241 & $0.621-2.479$ & 0.441 \\
\hline Plaques & 0.772 & $0.397-1.312$ & 0.286 \\
\hline
\end{tabular}

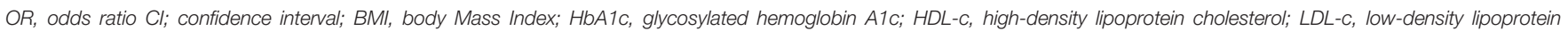
cholesterol; $E_{2}$, Estradiol; SHBG, sex hormone binding globulin.

The bold values indicates statistical significance.

TABLE 4 | An adjusted Logistic regression model between variables and hypogonadism status.

\begin{tabular}{|c|c|c|c|}
\hline Variable & OR & $95 \% \mathrm{Cl}$ & P Values \\
\hline Age & 1.008 & $0.985-1.033$ & 0.520 \\
\hline $\mathrm{BMI}$ & 1.149 & $1.033-1.279$ & 0.011 \\
\hline Fasting C-peptide & 1.009 & $0.738-1.379$ & 0.957 \\
\hline Triglycerides & 1.167 & $1.014-1.343$ & 0.031 \\
\hline Uric acid & 1.003 & 0.999-1.006 & 0.069 \\
\hline Osteocalcin & 0.965 & 0.899-1.036 & 0.321 \\
\hline$E_{2}$ & 0.986 & $0.977-0.996$ & 0.002 \\
\hline
\end{tabular}

OR, odds ratio Cl; confidence interval; BMI, Body Mass Index; $E_{2}$, Estradiol.

The bold values indicates statistical significance.

To explore the possible underlying mechanism for the phenomena, the risk factors for hypogonadism in male diabetic patients were explored in the overall population. Consistent with previous studies, majority of cases of hypogonadism in the two groups were hypogonadotropic hypogonadism $(23,26)$. This finding suggest that an inadequate release of gonadotropin is the main reason for low testosterone in diabetes patients. Our data showed that BMI was a pivotal risk factor for hypogonadism, consistent with previous studies reporting that male obesity is often accompanied with low testosterone and that BMI was negatively correlated to low testosterone in both T1DM and T2DM patients $(4,21,26)$. Low testosterone levels could aggravate fat depot, especially abdominal visceral fat, which is associated with the effects of testosterone on lipid storage, lipolysis and adipogenesis (27). On the contrary, increased visceral fat results in accumulation of proinflammatory cytokines and free fatty acids that aggravate insulin resistance and dysregulated leptin signaling could inhibit hypothalamic-pituitarygonadal (HPG) axis function $(28,29)$.

It is widely reported that the high expression of aromatase in the adipose tissue, which could increase the conversion of testosterone into estradiol, leading to decreased gonadotropin secretion (30). Our data have shown that estradiol was negatively associated with hypogonadism status in male diabetic patients after adjusting confounding factors. The findings were supported by a recent study reporting that lower aromatase expression in the adipose tissue of obese men were with low testosterone compared to controls with normal circulating testosterone (31). Moreover, our results further support the notion that although estradiol is important for this negative feedback, androgen receptor-mediated effects also play a role (32).

We also observed an association between uric acid and hypogonadism in our study consistent with the findings of other studies that uric acid was negatively correlated with testosterone levels in patients with $\operatorname{T2DM}(22,23)$. A previous study also reported decreased mean testosterone levels in patients with gouty kidney disease and gouty arthritis (33). It was reported that the relationship between uric acid and testosterone levels were bidirectional. On one hand, high levels of uric acid could form crystals in the testicular tissue leading to oxidative damage (34). On the other hand, low levels of testosterone decrease protein synthesis, which results in accumulation of endogenous purine causing hyperuricemia (35). However, after adjusting confounder factors, there was no association between uric acid and hypogonadism. Further studies are needed to clarify whether uric acid influences gonadotropin secretion.

In addition, triglycerides and total cholesterol were risk factors for hypogonadism in male diabetic patient. The results support the findings that men with hypogonadism may have 
dyslipidemia and testosterone treatment could significantly decrease total cholesterol and triglycerides $(6,30)$. Previous studies have reported that diabetic neuropathy was associated with the levels of testosterone in patients with T2DM (36). However, there were no association between hypogonadism status and microvascular or macrovascular complications of diabetes, and presence of plaques. In line with previous studies, fasting glucose, HbA1c levels and osteocalcin were not associated with hypogonadism in the present study (37-39).

This study has some limitations. First, the exclusion of patients with CKD may be a selection bias for this study; Second, the recruited patients were all inpatients with poor blood glucose or metabolic control, which might increase the risk of hypogonadism; Third, few men without diabetes as a proper comparison may bring about a confounding bias; Fourth, the sample size of patient with LADA was not adequate to detect significant differences. Despite these limitations, we believe that our study is valuable because our findings might not only contribute to further understand the effects of testosterone on glucose metabolism, but also help to better understand the effective way to prevention hypogonadism in male patients with diabetes.

\section{CONCLUSION}

In a population of requiring admission to a large urban hospital in China, the rate of hypogonadism was comparable to those of the T2DM group after adjusting confounding factors. The results suggested the association between hypogonadism and the etiology of hyperglycemia was limited in this study. BMI, triglycerides and estradiol were independently associated with the presence of $\mathrm{HH}$ in male diabetic patients.

\section{REFERENCES}

1. Liu B, Xiang Y, Liu Z, Zhou Z. Past, Present and Future of Latent Autoimmune Diabetes in Adults. Diabetes Metab Res Rev (2019) 18:e3205. doi: 10.1002/dmrr.3205

2. Buzzetti R, Zampetti S, Maddaloni E. Adult-Onset Autoimmune Diabetes: Current Knowledge and Implications for Management. Nat Rev Endocrinol (2017) Nov13(11):674-86. doi: 10.1038/nrendo.2017.99

3. Laugesen E, Østergaard JA, Leslie RD. Danish Diabetes Academy Workshop and Workshop Speakers. Latent Autoimmune Diabetes of the Adult: Current Knowledge and Uncertainty. Diabetes Med (2015) 32:843-52. doi: 10.1111/ dme. 12700

4. Kupelian V, Hayes FJ, Link CL, Rosen R, McKinlay JB. Inverse Association of Testosterone and the Metabolic Syndrome in Men Is Consistent Across Race and Ethnic Groups. J Clin Endocrinol Metab (2008) 93:3403-10. doi: 10.1210/ jc. 2008-0054

5. Dhindsa S, Prabhakar S, Sethi M, Arindam B, Chaudhuri A, Paresh D. Frequent Occurrence of Hypogonadotropic Hypogonadism in Type 2 Diabetes. J Clin Endocrinol Metab (2004) 89:5462-8. doi: 10.1210/jc.2004-0804

6. Corona G, Giagulli VA, Maseroli E, Vignozzi L, Aversa A, Zitzmann M, et al. Testosterone Supplementation and Body Composition: Results From a MetaAnalysis of Observational Studies. J Endocrinol Invest (2016) 39:967-81. doi: 10.1007/s40618-016-0480-2

7. Pessin JE, Saltiel AR. Signaling Pathways in Insulin Action: Molecular Targets of Insulin Resistance. J Clin Invest (2000) 106(2):165-9. doi: 10.1172/ JCI10582

\section{DATA AVAILABILITY STATEMENT}

The raw data supporting the conclusions of this article will be made available by the authors, without undue reservation.

\section{ETHICS STATEMENT}

The studies involving human participants were reviewed and approved by The Institutional Human Subjects Review Board of Shanghai Tenth People's Hospital. The patients/participants provided their written informed consent to participate in this study.

\section{AUTHOR CONTRIBUTIONS}

MZ: designed and wrote. MC, RC, and PY: performed and collected. JG, XC, and CS: participated in recruiting the patients. HS and SQ: edited. All authors contributed to the article and approved the submitted version.

\section{FUNDING}

This study is supported by grants from the National Key R\&D Program of China (No.2018YFC1314100) and the National Nature Science Foundation (No.81601269).

\section{ACKNOWLEDGMENTS}

This study would not have been possible without the participation of these patients.

8. Gianatti EJ, Grossmann M. Testosterone Deficiency in Men With Type 2 Diabetes: Pathophysiology and Treatment. Diabetes Med (2020) 37(2):17486. doi: 10.1111/dme.13977

9. Holt SK, Lopushnyan N, Hotaling J, Sarma AV, Dunn RL, Cleary PA, et al. Prevalence of Low Testosterone and Predisposing Risk Factors in Men With Type 1 Diabetes Mellitus: Findings From the DCCT/EDIC. J Clin Endocrinol Metab (2014) 99(9):E1655-60. doi: 10.1210/jc.2014-1317

10. Chandel A, Dhindsa S, Topiwala S, Chaudhuri A, Dandona P. Testosterone Concentration in Young Patients With Diabetes. Diabetes Care (2008) 31 (10):2013-7. doi: 10.2337/dc08-0851

11. Hawa MI, Thivolet C, Mauricio D, Alemanno I, Cipponeri E, Collier D, et al. Metabolic Syndrome and Autoimmune Diabetes: Action LADA 3. Diabetes Care (2009) 32(1):160-4. doi: 10.2337/dc08-1419

12. Mollo A, Hernandez M, Marsal JR, Esquerda A, Rius F, Blanco-Vaca F, et al. Latent Autoimmune Diabetes in Adults Is Perched Between Type 1 and Type 2: Evidence From Adults in One Region of Spain. Diabetes Metab Res Rev (2013) 29(6):446-51. doi: 10.1002/dmrr.2411

13. Maddaloni E, Moretti C, Del Toro R, Sterpetti S, Ievolella MV, Arnesano G, et al. Risk of Cardiac Autonomic Neuropathy in Latent Autoimmune Diabetes in Adults Is Similar to Type 1 Diabetes and Lower Compared to Type 2 Diabetes: A Cross-Sectional Study. Diabetes Med (2021) 38(2):e14455. doi: 10.1111/dme.14455

14. Zhang M, Sheng C, You H, Cai M, Gao J, Cheng X, et al. Comparing the Bone Mineral Density Among Male Patients With Latent Autoimmune Diabetes and Classical Type 1 and Type 2 Diabetes, and Exploring Risk Factors for Osteoporosis. J Endocrinol Invest (2021). doi: 10.1007/s40618-020-01472-6 
15. Fourlanos S, Dotta F, Greenbaum CJ, Palmer JP, Rolandsson O, Colman PG, et al. Latent Autoimmune Diabetes in Adults (LADA) Should be Less Latent. Diabetologia. (2005) 48:2206-12. doi: 10.1007/s00125-005-1960-7

16. Wu FC, Tajar A, Beynon JM, Pye SR, Silman AJ, Finn JD, et al. Identification Of Late-Onset Hypogonadism in Middle-Aged and Elderly Men. N Engl J Med (2010) 363(2):123-35. doi: 10.1056/NEJMoa0911101

17. Bhasin S, Brito JP, Cunningham GR, Hayes FJ, Hodis HN, Matsumoto AM, et al. Testosterone Therapy in Men With Hypogonadism: An Endocrine Society Clinical Practice Guideline. J Clin Endocrinol Metab (2018) 103 (5):1715-44. doi: 10.1210/jc.2018-00229

18. Kapoor D, Aldred H, Clark S, Channer KS, Jones TH. Clinical and Biochemical Assessment of Hypogonadism in Men With Type 2 Diabetes: Correlations With Bioavailable Testosterone and Visceral Adiposity. Diabetes Care (2007) 30(4):911-7. doi: 10.2337/dc06-1426

19. Yang W, Liu J, Shan Z, Tian H, Zhou Z, Ji Q, et al. Acarbose Compared With Metformin as Initial Therapy in Patients With Newly Diagnosed Type 2 Diabetes: An Open-Label, Non-Inferiority Randomized Trial. Lancet Diabetes Endocrinol (2014) 2(1):46-55. doi: 10.1016/S2213-8587(13)70021-4

20. Keelan JA, Mattes E, Tan H, Dinan A, Newnham JP, Whitehouse AJ, et al. Androgen Concentrations in Umbilical Cord Blood and Their Association With Maternal, Fetal and Obstetric Factors. PloS One (2012) 7(8):e42827. doi: 10.1371/journal.pone.0042827

21. Cooper LA, Page ST, Amory JK, Anawalt BD, Matsumoto AM. The Association of Obesity With Sex Hormone-Binding Globulin Is Stronger Than the Association With Ageing-Implications for the Interpretation of Total Testosterone Measurements. Clin Endocrinol (Oxf) (2015) 83(6):82833. doi: $10.1111 /$ cen. 12768

22. Li Y, Zhang M, Liu X, Cui W, Rampersad S, Li F, et al. Correlates and Prevalence of Hypogonadism in Patients With Early- and Late-Onset Type 2 Diabetes. Andrology. (2017) 5(4):739-43. doi: 10.1111/andr.12360

23. Cheung KK, Luk AO, So WY, Ma RC, Kong AP, Chow FC, et al. Testosterone Level in Men With Type 2 Diabetes Mellitus and Related Metabolic Effects: A Review of Current Evidence. J Diabetes Investig (2015) 6(2):112-23. doi: $10.1111 /$ jdi.12288

24. Wang Q, Kangas AJ, Soininen P, Tiainen M, Tynkkynen T, Puukka K, et al. Sex Hormone-Binding Globulin Associations With Circulating Lipids and Metabolites and the Risk for Type 2 Diabetes: Observational and Causal Effect Estimates. Int J Epidemiol (2015) 44(2):623-37. doi: 10.1093/ije/dyv093

25. Simó R, Sáez-López C, Barbosa-Desongles A, Hernández C, Selva DM. Novel Insights in SHBG Regulation and Clinical Implications. Trends Endocrinol Metab (2015) 26(7):3763-83. doi: 10.1016/j.tem.2015.05.001

26. Chillarón JJ, Fernández-Miró M, Albareda M, Vila L, Colom C, Fontserè S, et al. Age, Insulin Requirements, Waist Circumference, and Triglycerides Predict Hypogonadotropic Hypogonadism in Patients With Type 1 Diabetes. J Sex Med (2015) 12(1):76-82. doi: 10.1111/jsm.12748

27. Kelly DM, Jones TH. Testosterone and Obesity. Obes Rev (2015) 16(7):581606. doi: 10.1111/obr.12282.

28. Luukkaa V, Pesonen U, Huhtaniemi I, Lehtonen A, Tilvis R, Tuomilehto J, et al. Inverse Correlation Between Serum Testosterone and Leptin in Men. J Clin Endocrinol Metab (1998) 83(9):3243-6. doi: 10.1210/jcem.83.9.5134
29. Haffner SM, Miettinen H, Karhapää P, Mykkänen L, Laakso M. Leptin Concentrations, Sex Hormones, and Cortisol in Nondiabetic Men. J Clin Endocrinol Metab (1997) 82(6):1807-9. doi: 10.1210/jcem.82.6.3978

30. Wang C, Jackson G, Jones TH, Matsumoto AM, Nehra A, Perelman MA, et al. Low Testosterone Associated With Obesity and the Metabolic Syndrome Contributes to Sexual Dysfunction and Cardiovascular Disease Risk in Men With Type 2 Diabetes. Diabetes Care (2011) 34(7):1669-75. doi: 10.2337/dc10-2339

31. Ghanim H, Dhindsa S, Abuaysheh S, Batra M, Kuhadiya ND, Makdissi A, et al. Diminished Androgen and Estrogen Receptors and Aromatase Levels in Hypogonadal Diabetic Men: Reversal With Testosterone. Eur J Endocrinol (2018) 178(3):277-83. doi: 10.1530/EJE-17-0673

32. Russell N, Grossmann M. Mechanisms IN ENDOCRINOLOGY: Estradiol as A Male Hormone. Eur J Endocrinol (2019) 181(1):R23-43. doi: 10.1530/EJE-18-1000

33. Hurina NM, Korpacheva-Zinych OV, Shuprovych AA. Interrelations of Uric Acid Metabolism Indices With Insulin and Testosterone Levels in Men With Type 2 Diabetes. Fiziol Zh (2010) 56(6):93-9. doi: 10.15407/fz56.06.093

34. Gong J, Xie Q, Han Y, Chen B, Li L, Zhou G, et al. Relationship Between Serum Uric Acid Levels, Metabolic Syndrome, and Arterial Stiffness in Korean. Korean Circ J (2010) 40(7):314-20. doi: 10.4070/kcj.2010.40.7.314

35. Afsar B, Karaca H. The Relationship Between Insulin, Insulin Resistance, Parathyroid Hormone, Cortisol, Testosterone, and Thyroid Function Tests in the Presence of Nephrolithiasis: A Comprehensive Analysis. Cent Eur J Urol (2014) 67(1):58-64. doi: 10.5173/ceju.2014.01

36. Hernández-Mijares A, García-Malpartida K, Solá-Izquierdo E, Bañuls C, Rocha M, Gómez-Martínez MJ, et al. Testosterone Levels in Males With Type 2 Diabetes and Their Relationship With Cardiovascular Risk Factors and Cardiovascular Disease. J Sex Med (2010) 7(5):1954-64. doi: 10.1111/j.1743-6109.2010.01705

37. Mathis G, Thomas MC, Sianna P, Sharpe K, Richard JM, Sophie C, et al. Low Testosterone Levels Are Common and Associated With Insulin Resistance in Men With Diabetes. J Clin Endocrinol Metab (2008) 93(5):1834-40. doi: 10.1210/jc.2007-2177

38. Kirmani S, Atkinson EJ, Melton LJ 3rd, Riggs BL, Amin S, Khosla S. Relationship of Testosterone and Osteocalcin Levels During Growth. J Bone Miner Res (2011) 26(9):2212-6. doi: 10.1002/jbmr.421

39. Schwetz V, Gumpold R, Graupp M, Hacker N, Schweighofer N, Trummer O, et al. Osteocalcin Is Not a Strong Determinant of Serum Testosterone and Sperm Count in Men From Infertile Couples. Andrology. (2013) 1(4):590-4. doi: $10.1111 /$ j.2047-2927.2013.00095

Conflict of Interest: The authors declare that the research was conducted in the absence of any commercial or financial relationships that could be construed as a potential conflict of interest.

Copyright () 2021 Cai, Cui, Yang, Gao, Cheng, Sheng, Li, Sheng, Qu and Zhang. This is an open-access article distributed under the terms of the Creative Commons Attribution License (CC BY). The use, distribution or reproduction in other forums is permitted, provided the original author(s) and the copyright owner(s) are credited and that the original publication in this journal is cited, in accordance with accepted academic practice. No use, distribution or reproduction is permitted which does not comply with these terms. 